\title{
Smart Waste Collection Processes A Case Study about Smart Device Implementation
}

\author{
Michael Reiner Kamm \\ University of Liechtenstein \\ michael.kamm@uni.li
}

\author{
Johannes Schneider \\ University of Liechtenstein \\ johannes.schneider@uni.li \\ Jan vom Brocke \\ University of Liechtenstein \\ jan.vom.brocke@uni.li
}

\begin{abstract}
For decades the core processes of collecting waste have been unchanged. Through new IoT-technologies, advances in sensors, and data transfer technologies, data-driven smart waste collection processes will replace old inefficient collection processes. Causing a shift from fix collection intervals to collection on demand, supported by smart algorithms and innovative web-applications. However, implementing such ideas come along with some almost insurmountable challenges related to wireless data transfer, battery lifetime and IoT infrastructure. Therefore, the question arises of how to implement IoT solutions in such complex and challenging environments. In order to contribute to the existing research about smart cities and autonomous IoT devices, we implemented smart devices in glass containers, measuring filling level over several months. The research study's outcomes are test results, data analysis and a prototype implementation for a reengineered waste collection process. Furthermore, we identified main challenges and key issues which obstruct the implementation and spread of such smart city applications.
\end{abstract}

\section{Introduction}

Significant technological developments in sensors, microcontrollers and wireless data transfer technologies, empower the diffusion of intelligent devices and interconnected smart systems [6]. This idea is summarized in the term "Internet of Things" (IoT). In the future, billions of connected devices will track, measure and control most of our daily life's applications [8]. Through this development, new business areas and opportunities have emerged, summarized in diverse Smart City and Smart Factory concepts. From those emerging areas, there is a clear trend towards the utilization of autonomous end-node devices with sensing, computing and communication capabilities
[19]. Such autonomous end-node devices are often powered by a battery or an accumulator, which comes with challenges related to energy consumption. Therefore, such devices are connected via lowbandwidth, low energy consuming data transfer technologies. Out of that, additional challenges are arising, such as difficulties when performing updates. Thus, the dependency on a battery generates various consequent challenges.

At the same time IoT offers new opportunities, empowering us to set a sustainable footprint in the world [17]. Such new application fields are for example the optimization of waste collection processes. According to Pariatamby \& Tanaka [17] the global population will increase up to 9 billion in 2050. In addition, the urbanization is constantly expanding, leading to massive pressure on city infrastructures [1, 17, 20]. Moreover, as the global economy expands more people buy and use products, which will result in an increase in waste generation. More and more solid waste is generated and ultimately needs to be collected for recycling and disposal [17]. However, the current waste collection processes are reaching their limits $[16,18]$. With an exponential increase in citizens living closely together, inefficient waste collection processes must be reengineered.

Although there is consensus about the need for smart waste collection processes, there have been little publications on real-world case studies, evaluating test results and publishing real data. In order to contribute to the ongoing discussion about digital connectivity in future cities, we implemented smart devices in a real case scenario. This case is about implementing an IoT solution to connect glass containers. The solution consists in measuring filling levels and sending the collected data via a low-power transmission technology to an IoT cloud. In the IoT cloud the data is analyzed and used to generate recommendations for process improvements.

With this research paper we share our experience made in over three years of project involvement, implementing IoT applications in a challenging 
environment, and provide new ideas of how to tackle various upcoming challenges. Results and main learnings out of this study are not limited to the waste management sector but can also contribute to other application fields in Smart City and Smart Factory projects. Thereby, we want to contribute to the ongoing discussion about smart cities, low power end-node devices, digital connectivity, IoT implementations and IoT affordances.

Last but not least, we want to emphasize that waste is an inevitable byproduct of our civilization. A growing number of people causes an increasing amount of waste which needs to be collected, handled, and managed at the same time. Therefore, increased efficiency in the waste collection process is mandatory for our future.

The paper is structured accordingly. The following section summarizes the current status in academic research and gives an overview of wireless data transfer technologies. In the third section the use case is introduced, providing insights from current waste collection processes. After that, the designed solution is presented and the filling behavior of a sample of glass containers is analyzed. The main findings and challenges are formulated, described, and discussed in the final section.

\section{Related work}

\subsection{IoT}

Internet of Things (IoT) describes the idea of interconnecting all kind of physical objects through the internet [2, 5, 8]. According to Chen et al. [2] our physical world becomes one interconnected information system, offering new possibilities but also challenges. Kevin Ashton established the core idea of IoT already in 1999 [6]. Nowadays, many obstacles from the beginning have been solved. For instance, the size and costs of data transfer chips have drastically decreased [6]. New IoT technologies set the foundation for Smart City and Smart Factory concepts $[2,5,6,26]$. According to Flammini \& Sisinni [5], the target is to inquire, collect and analyze data about events and processes in order to ensure a sustainable use of resources, increase production efficiency and improve life in cities. However, connected IoT devices need reliable bidirectional signaling to enable communication, interaction, and data transmission between devices and gateways [8]. In this field a lot of challenges are arising, related to scaling, reliability, bandwidth, security and power consumption $[5,24]$. One example is the increase in batteries utilization and a consequent concern about the battery lifetime [4]. Therefore, choosing the right data transfer technology is essential.

\subsection{Data Transfer Technologies}

Battery powered IoT devices are limited in their operations by the capacity of their power supply [15]. Therefore, minimizing the energy consumption required for inquiring and transferring data is fundamental $[9$, 15]. According to Miorandi et al. [15] the need to replace batteries after a certain time, represents an enormous barrier to the widespread implementation of autonomous IoT applications. Moreover, battery technology is facing practical limits [7]. Therefore, recent research projects focus on developing alternatives by replacing batteries with energy harvesting and passive wireless sensor networks [15]. However, reducing the amount of energy needed to measure, transmit and receive data remains a critical concern [21]. Figure 1 presents an overview of wireless data transfer technologies.

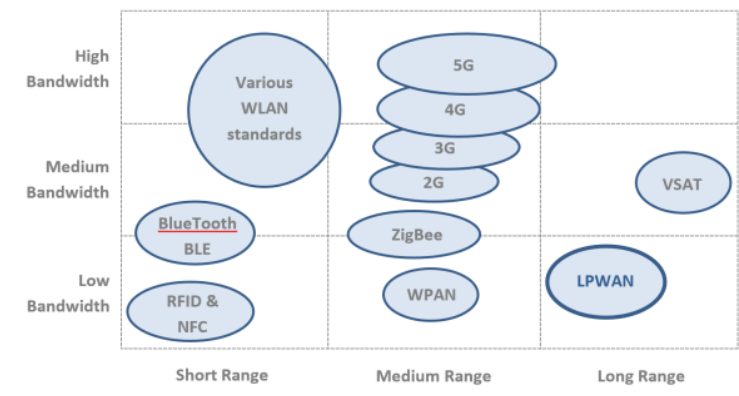

Figure 1: Overview of wireless data transfer technologies (Adapted from Egli [13])

Every wireless network technology has its strengths and weaknesses. While WLAN and telecommunication technologies such as $4 \mathrm{G}$ and $5 \mathrm{G}$ are optimized for transferring videos and stream large volumes of data in real time, they have a high energy consumption $[3,14$, 22]. Near-Field Communication (NFC) works with passive tags. These passive NFC tags work without a direct energy source [28]. Instead, these tags induce the needed energy from a part of the sender signal. Consequently, they are limited to the power of the magnetic field of the sender. The range lies between a few millimeters up to a few meters $[9,28]$.

2.2.1. LPWAN. Low Power Wide Area Networks (LPWAN) are especially interesting for IoT applications due to their very high geographical coverage and minimized energy consumption for data transmission $[21,25]$. The basic idea of LPWAN is a reduced bandwidth for the data transmission, since sensor units only send small data packages [25]. The range varies from a few kilometers in urban areas up to 15-30 
kilometers in rural areas [13]. The umbrella term LPWAN contains a variety of specific technologies, such as LoRa, NB-IoT and Sigfox.

2.2.2. LoRa. LoRa stands for "Long Range", and is a low power data transfer technology developed by Semtech [27]. LoRa's infrastructure is rolled out by several telecommunication companies, which are signed in the LoRa Alliance, as well as by private individuals or companies that want to establish their own IoT infrastructure. Since there is no necessary dependency to a network provider, LoRa offers a high flexibility for IoT applications [12, 13, 27]. LoRa makes use of the unlicensed frequencies (ISM bands) that are available worldwide. LoRaWAN is the MAC protocol for LoRa devices which optimizes the energy consumption and quality of service for the end-node devices [12, 13]. The LoRaWAN protocol is fully bidirectional, allowing data transfer in both directions. Furthermore, security solutions for end-to-end encryption and registration of end-node devices are included [27].

\subsection{Smart City}

Nowadays big Cities must deal with an exponentially increasing number of inhabitants living closely together. Therefore, cities must become more efficient and sustainable, work with less resources, reduce costs, ensure and enhance a high quality of life [15]. According to the China Academy of Information and Communications Technology (CAICT) [1], an increased urbanization in European and Chinese cities has led to energy and water scarcity, problems with waste management and disposal, and safety risks from ageing infrastructure. According to Sanseverino et al. [20] future cities are characterized by sustainable water generation, improved transportation, and efficient waste management (Figure 2).

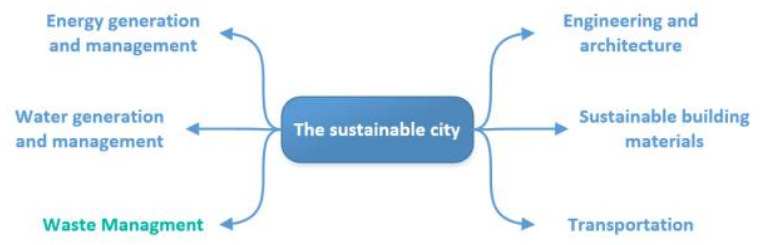

Figure 2: The sustainable city (adapted from Sanseverino et al. [20])

Many big projects in the past mainly focused on avoiding waste, offering new ways of packaging, enhanced recycling, and reusable technologies [11]. Nevertheless, the main issue is not only waste generation but also collecting waste in an efficient way $[10,17]$. According to Pariatamby \& Tanaka [17] increasing waste volumes become a major cause for health and environmental problems. Interestingly, the waste collection process itself did not change nor did it improve much over the last decades [10, 11, 17]. The current situation in many municipalities is characterized by emptying every container, no matter if it is full or not.

\subsection{Current solutions and projects}

There are various smart waste management projects executed worldwide in rural and urban areas [1, 20]. Recent solutions are based on different techniques and data transfer technologies. The product portfolio of vendors in this market differs in the service they offer. Some are offering new designed containers with integrated solutions, others offer sensor units which can be applied to an existing system. Most of these solutions make use of GSM technology. However, the use of GSM results in certain challenges, such as the dependency to a network provider and higher energy consumption in transferring data compared to LPWAN technologies (Figure 1) [3, 4, 13]. Some vendors share some commonalities, for example most sensor units are equipped with an ultrasonic sensor for measuring the filling level of a container. Most systems are powered by a battery or accumulator. The architecture and software solutions differ mainly on categorical choices such as: choice of data transfer technology (e.g. Lora vs. GSM vs. Sigfox...), usage of communication protocols, and the energy supply (battery powered or in combination with a solar panel) [23].

\section{The current situation in the case city}

The following case description was derived during the project work, in over three years of development and implementing a smart solution for glass containers in a small city in Austria. Therefore, a collaboration with various stakeholders such as municipalities, the responsible governments, waste truck drivers, the respective state environment agency, several waste collection service providers, and various smart city initiatives was established. Information was derived through first-hand experience, meetings, interviews and intensive observations, leading to a wide and in depth understanding of processes, regulations, cost structures, special boundary conditions, and challenges. Single processes, technologies, and operations might differ in other cities and countries. Nevertheless, the presented case provides insights in current processes and challenges for waste collection services.

The current situation in the case city is characterized by emptying every glass container in fixed intervals, no matter if it is full or not. The collection process itself is 
mainly planned based on experience of the collection companies and the drivers. There is no information available about the filling level of glass containers. Glass containers are scattered everywhere in cities and villages with their highest concentration at public places, such as main shopping areas, as well as in parks and along main roads. Municipalities are selfresponsible to organize the waste collection. Often the containers and their locations are provided by the municipality, but the collection is done by a third-party provider. High distances, huge weight, maintenance and repair costs, tons of consumed fuel, and various other expenses, make the collection processes very complex and expensive. From an economic and environmental perspective, the current collection process is neither efficient nor sustainable.

Identified main costs are:

- Waste collection vehicle costs

- Office and labor costs

- Employees (workforce and gross salaries)

- Maintenance

- Fuel expenses (high distances)

- Taxes, for e.g. waste collection vehicles

- Safety trainings

External factors causing unpredictable costs:

- Traffic jams

- Traffic lights

- Accidents

- Health of employees (many sick leaves)

The paradox: during waste collection even more waste is actually generated in terms of resource consumption such as maintenance effort, fuel consumption, and material usage.

In the use case containers are scattered at a great distance from each other. Waste collection vehicles drive between $100 \mathrm{~km}$ up to $500 \mathrm{~km}$ per day. Some collection vehicles drive up to $110.000 \mathrm{~km}$ per year. Highly corrosive fluids and poisonous waste decompose the vehicle and represent a health threat for employees. These corrosive fluids, the enormous weight of the truck, and the weight of the collected glass result in a high maintenance effort. Therefore, a collection vehicle does not last longer than seven years and the wheels need to be changed twice a year. In addition, the topographical challenges of every city are different. Hills, valleys, road management, waiting time due to traffic jams, traffic lights, and accidents end in an increase in time, fuel, and effort needed to collect the glass.

The identified root of all the above-mentioned inefficiencies is the lack of information. Missing information about the filling level of glass containers makes the entire processes inefficient and leads to unsustainable decisions based on experience.

\section{Designed solution and implementation}

The smart waste collection solution consists in attaching ultrasonic sensor units, capable of measuring waste levels in public glass containers. Figure 3 illustrates the data capturing process and transmission to the IoT cloud. In the cloud, analytical techniques are used to optimize routes, study the filling behavior, and build predictive models.

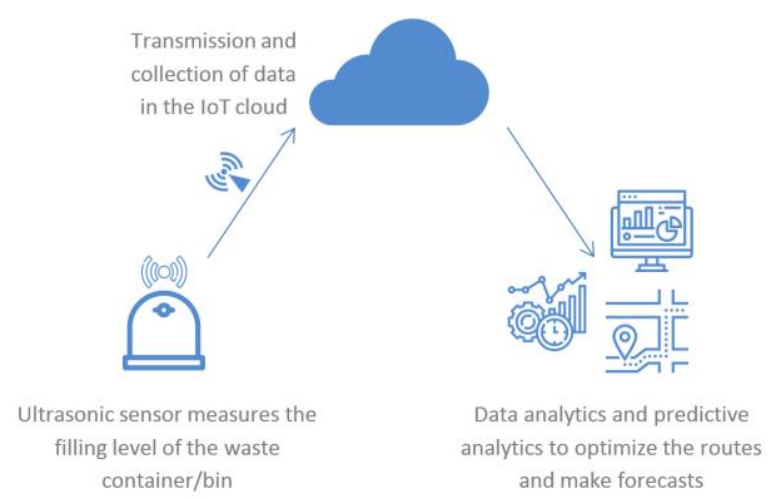

Figure 3: Data capturing and transmission to the IoT-cloud

\subsection{Hardware}

4.1.1. Sensor unit. The sensor unit consists of several parts, containing a microcontroller, an ultrasonic sensor, a real time clock, a battery, a LoRa chip, and housing.

Sensor: An ultrasonic sensor measures the filling level of containers. This sensor emits ultrasonic waves and measures the time until it detects the echo reflected from an object.

Lora chip: The LoRa chip enables low power consuming, long range data transmission and is one LPWAN technology. The LoRa chip communicates with a LoRa Gateway in a range of up to $15 \mathrm{~km}$ [12]. The gateway itself is then connected via a broadband internet connection to the IoT-Cloud.

Battery: The battery provides energy for all actions. Consequently, the lifetime and functionalities of the autonomous sensor unit are limited to the capacity of the battery. Therefore, it is important to implement low power consuming hardware and to schedule deep-sleep modes. Sleep modes enable energy savings when a device is not measuring, processing nor sending data.

4.1.2. IoT infrastructure. In order to be independent of telecommunication providers, an own IoT network 
infrastructure has been installed in collaboration with a local smart city initiative. The chosen technology is based on LoRa with several implemented gateways in the city.

\subsection{Software}

Based on the previously described technologies we implemented a platform to manage the installed sensors and their measured data over time. The implementation itself can be divided into three main parts as shown in Figure 4: the sensor application which is responsible for the measurement of the filling level inside waste containers, The Things Network ${ }^{1}$ (TTN) service which provides an open network to build IoT applications using LoRaWAN infrastructure, and the smart waste web application.

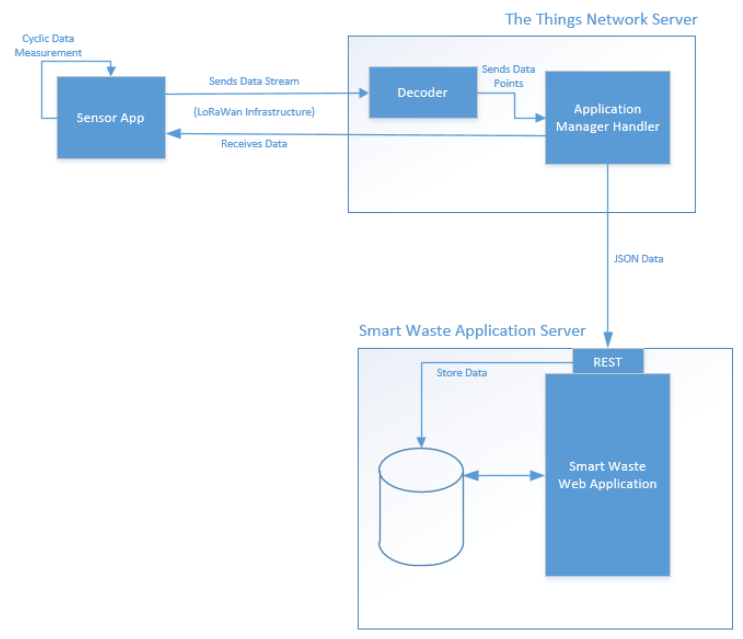

Figure 4: The overall software architecture

4.2.1. Data measurement. The sensor unit is based on a special Arduino ${ }^{2}$ board called Talk2 Whisper Node, designed for ultra-low power LoRaWAN applications. The sensor software running on an Atmel AVR ATMega328p is written in $\mathrm{C}$ and sends in a configurable cycle the measured distance of the supersonic sensor to a LoRaWAN gateway. For the data transmission, the measured distance (in millimeter) is scaled to a 2-byte value. Simultaneously, the configured cycle time (in minutes) is also transformed into a 2-byte value and sent with the same byte stream.

The sensor unit can also receive data. Therefore, we implemented a function to adjust the cycle time for the distance measurement, as well as some additional debug functions. The cycle time can be adjusted between half a minute up to several years. The TTN service is used to

\footnotetext{
${ }^{1}$ https://www.thethingsnetwork.org/

${ }^{2}$ https://www.arduino.cc/
}

get this data into the web application. TTN offers for registered users an open and free service to host own and use other LoRa gateways and services. A TTN registered gateway can receive the data sent by a sensor and forwards the data using an internet connection to the TTN server. Depending on the configuration of the packet forwarder MQTT or gRPC can be used. To identify which sensor belongs to which server application special keys and identifiers are used. The server receives the data and decodes the byte stream accordingly. Whenever the server receives new data, it is forwarded to our web application. For this purpose, a REST API is provided by the smart waste application.

4.2.2. Smart waste application. The smart waste application is implemented in Python using the Django ${ }^{3}$ web framework. The measurement values and cycle times are stored in a MySQL database. New mounted sensors are registered via the provided REST API.

The administrator of the smart waste application can group various sensors to a network. Figure 5 shows one of these networks containing three sensors.

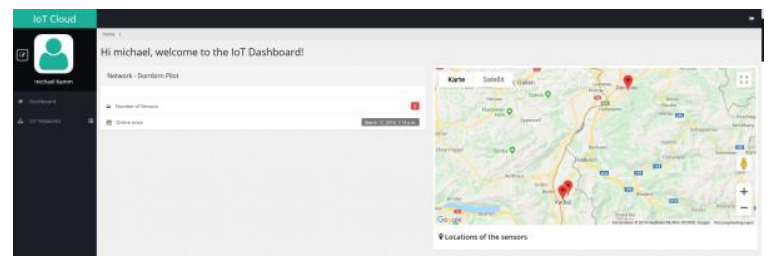

Figure 5: Sensor network overview

By clicking on a specific network, the single sensors can be selected, and the sensor specific data are shown. Figure 6 illustrates the location information and the measurement values in millimeters over time.

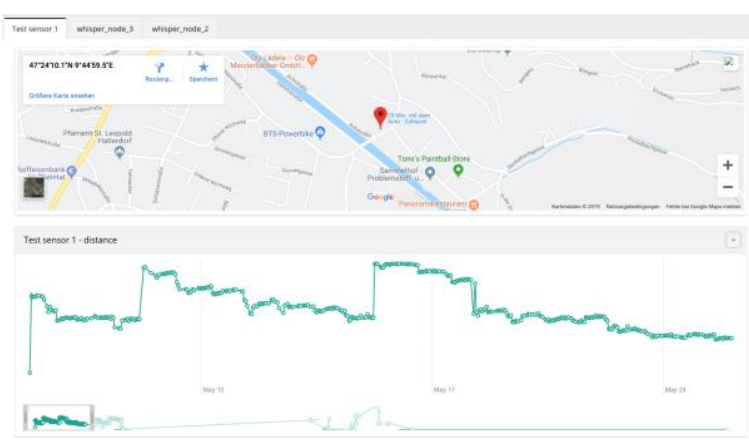

Figure 6: Filling level of a single sensor

With the help of a slide bar, specific time intervals can be selected. In this way, additional details about the measurement can be displayed.

${ }^{3}$ https://www.djangoproject.com/ 


\section{Data Analysis}

The following section contains the results of the first prototype implementation, revealing first insights in filling behavior of different glass containers. For this purpose, five glass containers were equipped with smart sensor units for a duration of several months. The following figures present sample data about the filling behavior of glass containers.

\section{Characteristics of glass container A:}

Location: residential area

Emptying interval: every Tuesday

Sensor measurement interval (cycle time): $60 \mathrm{~min}$ Period: 14.05.2018 - 20.05.2018

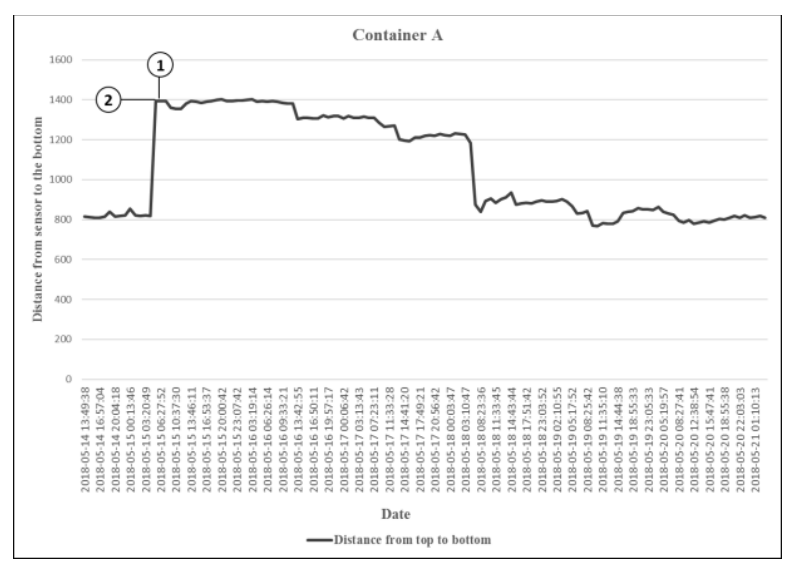

Figure 7: Sensor measurements container A

The inverse function of the distance shows the filling level, illustrated in Figure 8.

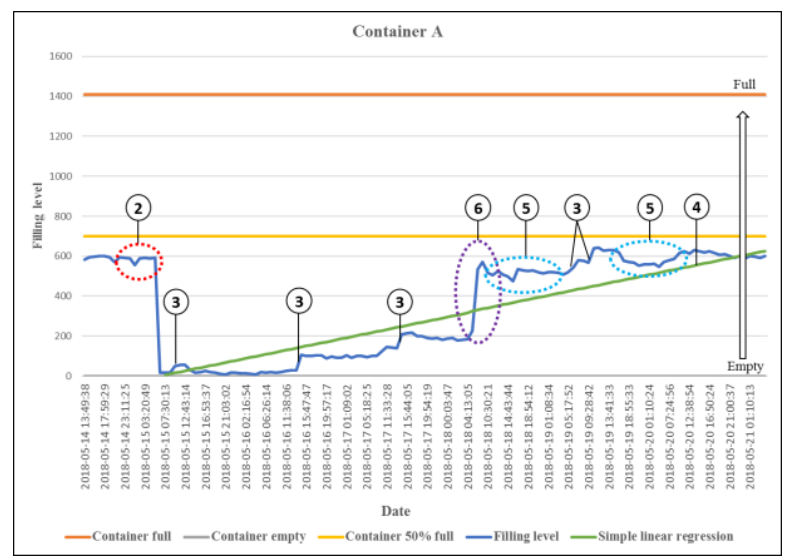

Figure 8: Filling behavior container $\mathrm{A}$

Out of the data, the following insights can be drawn:

1) The glass container is $1400 \mathrm{~mm}$ deep (Figure 7).

2) Emptying intervals are indicated from the data (Point 2 in Figure 7 \& Figure 8). In this case, Tuesdays around $7.00 \mathrm{am}$. The container was not even $50 \%$ filled when it was collected on the 15.05.2018. Even more interesting is the fact, that one week later, on the 21.05 .15 (next emptying cycle), the filling level is approximately on the same level.

3) Glass quantity thrown away by a single person.

4) The slope can be calculated, indicating the trend and the filling behavior of a container. Based on this information, further predictions can be made. For example: in 7 days $600 \mathrm{~mm}$ glass were filled in the container. That equals $86 \mathrm{~mm}$ per day on average. With the assumption of a linear filling behavior, the container will be full in around 16 days (remark: emptying cycles are every seven days). Obviously, a multi-linear regression, tree or logistic function will predict the filling level even more accurately.

5) Small drop downs in the graph are due to the fact, that when throwing glass in a container, a pile of glass is formed in the container's center. At a certain point, when more glass is thrown in the container, the pile falls to the side. Point 5 shows the effect of sliding glass to the side, when the next person throws in glass.

6) A significantly higher amount of glass was thrown in the container - approximately five times more than an average person. An assumption can be drawn, that glass bottles from a restaurant or a cocktail bar were thrown in the container or the start of the holiday season in Austria had an effect (18.05.18 start of the Pentecost holidays).

\section{Characteristics of glass container B:}

Location: city center

Emptying interval: every Tuesday

Sensor measurement interval (cycle time): varied between $1 \mathrm{~min}-6 \mathrm{~h}$

Height: $1200 \mathrm{~mm}$

Period: 12.10 .2018 - 29.10.18

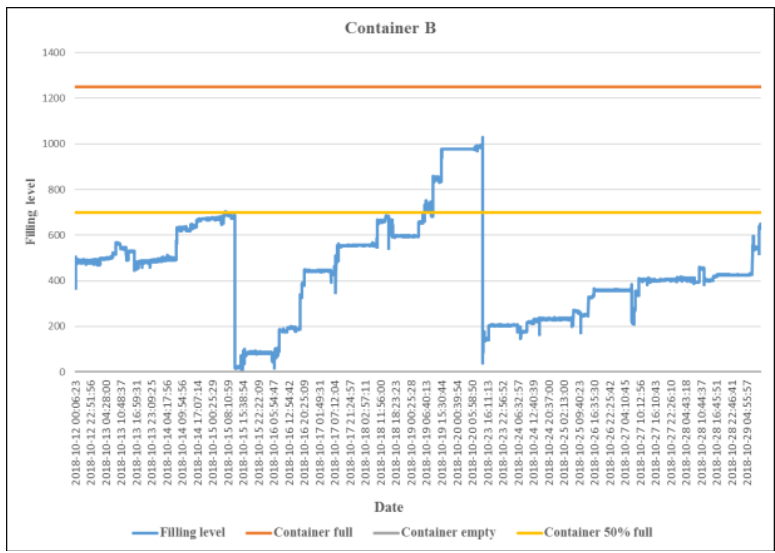

Figure 9: Filling behavior container B

Figure 9 illustrates that the filling behavior of containers can be different from week to week. 


\section{Characteristics of glass container $\mathbf{C}$ :}

Location: outside of the city

Emptying interval: every second Thursday

Sensor measurement interval (cycle time): varied between $1 \mathrm{~min}-6 \mathrm{~h}$

Height: $1400 \mathrm{~mm}$

Period: 11.04.2019 - 08.05.2019

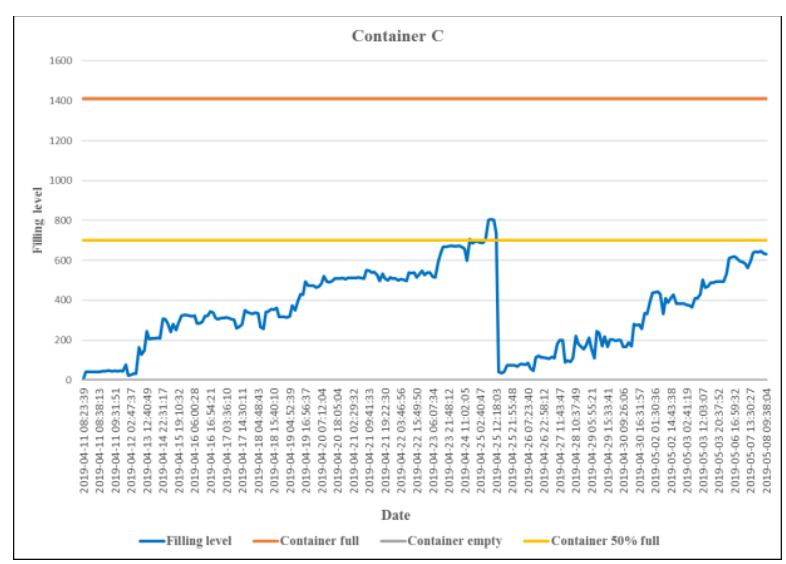

Figure 10: Filling behavior container $C$

In the drawn sample every container has a very different filling behavior. While container A has a filling rate of approximately once in two weeks, container B is approximately full after a week. Container $\mathrm{C}$ is approximately full every four weeks. To conclude, all containers in our sample were not full when they were collected.

\section{Main findings out of the case study}

During the development and implementation phases of the smart waste collection solution, several challenges were faced. Addressing these challenges resulted in a list of lessons learned:

1) The difference between battery powered IoT devices and devices with unlimited energy supply These two cases are fundamentally different. Use cases where battery powered sensor units are implemented, face a number of additional challenges. There is a dilemma between the limited battery capacity and the high energy consuming wireless data transfer. The limited energy resource restricts the implementation of many IoT ideas developed in theory. Supporting the work of Sheikh und Mahmoud, reducing the amount of energy needed to measure, process, transmit and receive data, is of great importance [21]. In case of battery powered IoT devices, the following two sub-challenges are observed:

\section{a. IoT infrastructure}

A fundamental question to ask when planning or selecting an IoT infrastructure is: "How to get the data as efficiently as possible from the end-node device to the IoT cloud?". There are several constraints that need to be taken into account: topography of the city or village, network coverage and penetration, bandwidth, data transfer rate and latency, distance, and energy consumption for data transmission.

\section{b. Update problem}

Bidirectional communication between the sensor unit and the application is essential but limited to the capabilities of the data transfer technology. Since sensor data consist out of a few bytes, often low band width data transfer technologies for energy efficient transmission are in place. Consequently, heavy software updates of the distributed sensor units, via this low bandwidth data transfer technologies, cause problems or are not possible to execute. Thus, once implemented, autonomous IoT devices cannot be updated with large updates. This needs to be considered before the widespread implementation of autonomous IoT devices.

\section{2) Providing an end to end solution}

Embedding a newly developed IoT solution within a legacy system, combining various technologies, platforms, and integrating the new processes, represents a tremendous effort. Being the intermediary between several stakeholders, such as municipalities and thirdparty providers, intensifies the problematic. Various interests, viewpoints, and decision processes are hard to unite, and are very time consuming. Therefore, IoT projects in cities go beyond technical development and touch all kinds of different fields.

\section{3) Dependencies}

Dependence on a network or data transfer infrastructure provider has advantages but also has its risks. For example, in case of limited network coverage, a new Io T infrastructure is only rolled out when the network provider has an incentive to do so. Some areas of the village or city may remain uncovered. Another challenge for the realization of smart city projects is the dependency on municipalities and their decision processes. Decision making processes differ from one municipality to another in time needed, participating stakeholders and involved state agencies.

\section{4) There is no uniform solution}

There is no one-fits-all solution. Hardware and software often need to be adapted to a large extent in every IoT project. Every boundary condition has an impact and shapes the solution. Every application requires a certain extend of customization and engineering.

5) Organizational inabilities to manage IoT complexities of the end-user

The customer or end user often does not have the required skill set to develop and host an IoT solution. Therefore, they are dependent on a third-party provider. 
In the specific case of the smart waste collection project, the stakeholders do not have the necessary competences. Since there is a lack of software and hardware engineers, as well as data analysis capabilities, a permanent collaboration with a third-party provider is necessary.

\section{6) Cost-benefit ratio is critical}

At every step of the development, the cost-benefit ratio needs to be considered. IoT projects need an initial investment in hardware procurement and development, as well as in software development. High initial investment costs obstruct the implementation of IoT solutions. Therefore, the right implementation strategy is essential.

\section{7) Sustainable development}

New IoT technologies empower us to improve areas that have not been improved for decades. A good example is the waste management sector, gathering filling levels and adapting inefficient routes is critical for leaving a green footprint. However, with billions of connected devices, the consumed energy for processing and sending data will also have a negative impact. Especially in the case of battery powered sensor units, the energy consumption is critical. Who wants to exchange billions of batteries from time to time? The need to exchange batteries obstructs the widespread implementation of autonomous IoT sensor units.

\section{Discussion}

The various software, hardware, and management problems from chapter 6 were addressed during the project work. The following chapter presents insights into the way certain challenges and topics were addressed:

1a) \& 3) As described in chapter 4, an own IoT network infrastructure based on LoRa was build up in collaboration with a local smart city initiative. Thus, there was no dependency to a network provider and data was transferred with no additional transfer costs to the cloud. The sensor units were remaining in a deep sleep mode when they were inactive. An efficient bidirectional communication was executed with byte streams. For example, the cycle time (measurement interval of the sensor) can be addressed with the byte stream (downlink) $03003 \mathrm{C}$ (hexadecimal). 03 is the identifier for a commando code. $3 \mathrm{C}$ equals the decimal number of 60 . A downlink of a byte stream with the values $0300 \mathrm{~F} 0$ would therefore adjust the cycle time to $240 \mathrm{~min}$. This cycle time can be adjusted from half a minute up to years. The encoding and decoding follows a defined scheme. In case of changes in the scheme, it needs to be changed on every single device. Furthermore, the LoRaWan protocol Class A queues downlinks, and only executes them with an incoming uplink (sensor unit sends data). This ensures that the sensor unit can stay as long as possible in deep sleep mode. Once it wakes up for measuring and sending the data, the downlink is executed simultaneously. Further challenges are related to communication issues and data loss.

1b) \& 7) In order to address the update problematic, we preprogrammed special functions which might be needed in the future. One of these functions we called the "self-adjusting function". The self-adjusting function can be addressed through a command in the downlink. The idea: the sensor pre-calculates the filling behavior of the glass container based on historical data, stored directly on the sensor unit. With this information the sensor unit adjusts the cycle time itself and decides when to wake up for the next measurement and data transfer. This function can be extended so that the sensor sends data only when it is appropriate or important. Therefore, the sensor unit can remain in the deep sleep mode as long as possible. In consequence, the batterie lifetime can be extended, since waking up, sending and receiving data consumes a lot of energy.

2) Working strongly together with all stakeholders from the beginning was extremely important already in the developing phase. Therefore, we collaborated with the respective environment state agency, collection services municipalities, and other smart city initiatives. Change management and willingness to adapt new processes from all sides are fundamental.

4) \& 6) For the development of hardware and software an incremental design approach is necessary. Not every idea can be implemented in practice. Especially in the beginning of an IoT project, only the most essential hardware parts and functionalities should be considered. For the waste collection case, it is essential to use hardware designed for this purpose. Standard hardware boards are not suitable due to high energy consumption. Software and hardware were designed for the specific waste collection case and adapted to the special boundary conditions. In terms of cost-benefit ratio, a standard ultrasonic sensor can be used, since there is no need for an exact measurement. It is more about the tendencies, and if a container is $20 \%$ or $60 \%$ filled. Our suggestion for successfully implementing IoT projects: start small in a representative test environment and scale step by step.

\section{Implications}

Based on the data about filling levels, advanced route optimization and further services can be offered. 
In addition, new business- fields and markets can be created. Arising opportunities are:

- Advanced route optimization and highly adaptive and flexible routes

- Individual emptying cycles per container based on filling behavior

- Waste collection on demand

- Prioritizing full containers in the emptying cycle

- Basic emptying cycles to avoid smell (depending on temperature)

- Creation of "waste maps"

- Smart Bin Placement Management

- Complete change of existing pricing and business models of waste collection services

Data driven smart waste collection is characterized by highly adaptive routes, considering the individual filling behavior of each container. Full containers are prioritized in the emptying cycle (Figure 11).

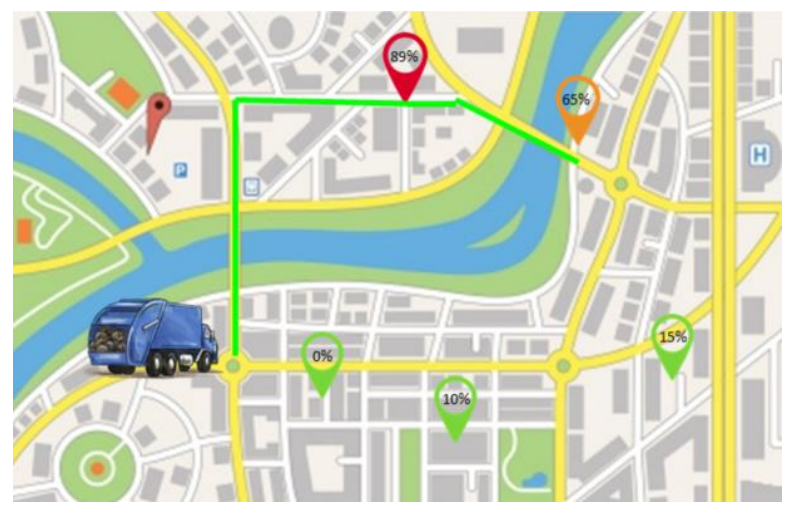

Figure 11: Adaptive collection routes

In addition, the creation of "waste maps" can help to detect problem areas of a city, where the containers are often overfull and as a result waste is lying around. This problem can be tackled through "Smart Bin Placement Management". In such areas, additional containers or containers of varying sizes could be installed. A container with a very frequent filling behavior can be replaced with a bigger container or by placing a second or a third one close. The ability to rearrange the amount of glass containers in certain locations allows waste collection services to manipulate and influence how often they have to approach certain locations to pick up the waste. Future opportunities are offering improved services, such as predictive analytics, and advanced route optimization. Several new variables and information, such as distance between containers, filling level, filling behavior, weather information, traffic data, holidays and events can be considered in the adaptive route planning algorithms.

We claim, that the change to data driven processes can lead to a:
- Decrease in the number of employees of waste collection services

- Decrease in overall workload and working hours

- Decrease in maintenance and operating expenses for waste collection vehicles

- Decrease in fuel consumption and $\mathrm{CO}_{2}$ emissions

- Cleaner cities due to less overfilled containers

- Sustainability and environmental friendliness

- Increase in transparency about waste generation and improved communication between municipalities

Currently, many municipalities are self-responsible for their waste collection processes or assign third-party providers. The newly generated data about filling levels and filling behavior, the optimization of inefficient collection processes, generation of waste maps and Smart Bin Placement management allow for the improvement of the entire waste management supply chain and offer municipalities an improved way of working closer together.

\section{Conclusion}

For decades the core processes of collecting waste have been unchanged. One of the major underlying issues is the missing information about filling level and filling behavior of containers. New IoT technologies can provide a solution. However, their implementation is concerned with several almost insurmountable challenges. In this research paper we contribute to the ongoing discussion about digital connectivity in future cities by:

A) A real-world case study and the implementation of sensor units in a challenging smart city application field.

B) Sharing our experience from over three years of project involvement and summarizing the main findings.

C) Giving an overview of the current glass container collection processes.

D) Evaluating first results and publishing real data and therefore giving preliminary insights into the filling behavior of glass containers. These insights support the claim that current collection processes based on fix intervals are neither efficient nor sustainable.

E) Sharing our ideas for improving the waste collection process, in order to establish data driven collection processes.

Main findings and learnings out of this study are not limited to the waste sector, but also contribute to other IoT application fields with similar challenges and obstacles. Regarding waste management, our mindset 
must change. Every single piece of plastic, every single piece of paper has a certain value. It is not acceptable that waste pollutes oceans and landscapes, with dramatic consequences on the environment. Increasing the efficiency of waste collection processes is therefore mandatory. With this publication we want to make our contribution towards changing the inefficient waste collection process, in order to leave a green footprint for the next generations.

\section{References}

[1] CAICT, Comparative Study of Smart Cities in Europe and China 2014, 1st edn., Springer Berlin Heidelberg, Berlin, Heidelberg, s.1., 2016.

[2] Chen, L., M. Tseng, and X. Lian, "Development of foundation models for Internet of Things", Frontiers of Computer Science in China, 4(3), 2010, pp. 376-385.

[3] Cox, C., An Introduction to LTE: LTE, LTE-Advanced, SAE and 4G Mobile Communications, 2nd edn., John Wiley \& Sons, Hoboken, 2012.

[4] Fagas, G., ed., ICT - Energy - Concepts Towards Zero Power Information and Communication Technology, InTech, 2014.

[5] Flammini, A. and E. Sisinni, "Wireless Sensor Networking in the Internet of Things and Cloud Computing Era", Procedia Engineering, 87, 2014.

[6] Gubbi, J., R. Buyya, S. Marusic, and M. Palaniswami, "Internet of Things (IoT): A vision, architectural elements, and future directions", Future Generation Computer Systems, 29(7), 2013, pp. 1645-1660.

[7] Itoh, T., RF Technologies for Low Power Wireless Communications, 1st edn., Wiley-IEEE Press, S.1., 2001.

[8] Jankowski, S., J. Covello, H. Bellini, J. Ritchie, and D. Costa, IoT primer: The Internet of Things: Making sense of the next mega-trend, 03 September 2014.

[9] Lee, I. and K. Lee, "The Internet of Things (IoT): Applications, investments, and challenges for enterprises", Business Horizons, 58(4), 2015, pp. 1-10.

[10] Levi, A., ed., 5th International Conference on New Technologies, Mobility and Security (NTMS), 2012: 7 - 10 May 2012, Istanbul, Turkey, IEEE, Piscataway, NJ, 2012.

[11] Llatas, C. and M. Osmani, "Development and validation of a building design waste reduction model", Waste management (New York, N.Y.), 56, 2016, pp. 318-336.

[12] http://www.semtech.com/wireless-rf/internet-ofthings/lora-applications/briefs, accessed 6-7-2019.

[13] https://www.slideshare.net/PeterREgli/lpwan, accessed 6-7-2019.

[14] Mavromoustakis, C.X., G. Mastorakis, and J.M. Batalla, eds., Internet of Things (IoT) in 5G Mobile Technologies, Springer International Publishing, Cham, s.l., 2016.
[15] Miorandi, D., S. Sicari, F. Pellegrini, and I. Chlamtac, "Internet of things: Vision, applications and research challenges", Ad Hoc Networks, 10(7), 2012, pp. 1497-1516.

[16] Morrissey, A.J. and J. Browne, "Waste management models and their application to sustainable waste management", Waste management (New York, N.Y.), 24(3), 2004, pp. 297-308.

[17] Pariatamby, A. and M. Tanaka, Municipal solid waste management in Asia and the pacific islands: Challenges and strategic solutions, Springer, Singapore, 2014.

[18] Pasang, H., G.A. Moore, and G. Sitorus, "Neighbourhood-based waste management: a solution for solid waste problems in Jakarta, Indonesia", Waste management, 27(12), 2007, pp. 1924-1938.

[19] Rane, U.V., V.R. Gad, R.S. Gad, and G.M. Naik, "Reliable and Scalable Architecture for Internet of Things for Sensors Using Soft-Core Processor", in Internet of Things, Smart Spaces, and Next Generation Networks and Systems, 2015. Springer International Publishing: Cham.

[20] Riva Sanseverino, E., R. Riva Sanseverino, V. Vaccaro, and G. Zizzo, Smart Rules for Smart Cities: Managing Efficient Cities in Euro-Mediterranean Countries, Springer International Publishing, Cham, s.1., 2014.

[21] Sheikh, O.M. and S.A. Mahmoud, Cross-Layer Design for Smart Routing in Wireless Sensor Networks, INTECH Open Access Publisher, 2012.

[22] Shorey, R., ed., Mobile, wireless, and sensor networks: Technology, applications, and future directions, IEEE Press, Piscataway, 2006.

[23] Shukla, B., S.K. Khatri, and P.K. Kapur, eds., 2016 5th International Conference on Reliability, Infocom Technologies and Optimization (ICRITO) (Trends and Future Directions): September 7-9, 2016, Amity University Uttar Pradesh, Noida, India, IEEE, 2016.

[24] Sicari, S., A. Rizzardi, L.A. Grieco, and A. CoenPorisini, "Security, privacy and trust in Internet of Things: The road ahead", Computer Networks, 76, 2015, pp. 146164 .

[25] Suhonen, J., Low-power wireless sensor networks: Protocols, services and applications, Springer, New York, NY u. a., 2012.

[26] Vesco, A. and F. Ferrero, eds., Handbook of research on social, economic, and environmental sustainability in the development of smart cities, Information Science Reference an imprint of IGI Global, Hershey, Pa, 2015.

[27] http:/www.semtech.com/wireless-rf/internet-ofthings/what-is-lora/, accessed 6-7-2019.

[28] Yan, L., Y. Zhang, L.T. Yang, and H. Ning, eds., The Internet of things: From RFID to the next-generation pervasive networked systems, Auerbach Publications, New York, 2008. 